\title{
Vom angedrohten Grexit zur differenzierten Integration
}

von Fritz W. Scharpf

In der Schlussphase des Verhandlungsmarathons über das vorläufig letzte griechische Hilfspaket hat der angedrohte Grexit die bedingungslose Kapitulation der Tsipras-Regierung erzwungen. Aber schon jetzt ist klar: Damit hat Wolfgang Schäuble zwar die Schlacht, nicht aber den Krieg um den Euro gewonnen. Im Gegenteil, mit der Härte der Kapitulationsbedingungen hat Deutschland ein Euro-Regime zementiert, das Europa ökonomisch zerreißt, und das sich deshalb politisch kaum durchhalten lässt.

\section{Das „deutsche Modell“ profitiert vom Euro}

Im Vergleich zu anderen europäischen Ländern hatte die exportorientierte deutsche Wirtschaft seit den frühen fünfziger Jahren von stabilem Geld, mäßigen Lohnsteigerungen und niedrigen Inflationsraten profitiert. Aber nach dem Zerfall des Bretton-Woods-Regimes fester Wechselkurse hatten allfallige Auf- und Abwertungen verhindert, dass die Export-Vorteile dieses deutschen Modells in den Himmel wachsen konnten. Dies änderte sich erst mit der Währungsunion, die weitere Auf- und Abwertungen unter ihren Mitgliedern ausschloss.

Nun erst konnte sich die - durch steigende Arbeitslosigkeit und zu hohe Realzinsen noch verstärkte - Sonderentwicklung der niedrigen deutschen Lohnstückkosten in vollem Maße auf den realen Wechselkurs auswirken (Abb. 1). Im Ergebnis entsprach sie seit der Mitte des Jahrzehnts einer mehr als 15\%igen Subvention deutscher Exporte. Die Folge war ein in der deutschen Wirtschaftsgeschichte einmaliger Anstieg der Exporte von etwa 25\% des Bruttoinlandsprodukts (BIP) auf den - für Länder von der Größe Deutschlands völlig ungewöhnlichen - Anteil von mehr als $45 \%$ (Abb. 2).Und da die Importe bei mäßiger Konsum- und Investitionsneigung im Inland nicht im gleichen Maße zunahmen, führten kumulative Leistungsbilanzüberschüsse auch zu einem ähnlich steilen Anstieg der deutschen Gläubigerposition gegenüber dem Ausland (Abb. 3). 
Beides, den extrem hohen Exportanteil und den Anstieg der NettoAuslandsguthaben verdankt Deutschland dem Euro. Zugleich ist die deutsche Wirtschaft damit jedoch extrem abhängig geworden von der globalen Nachfrage nach deutschen Investitionsgütern und deutschen Autos, und unsere Gläubigerposition wäre gefährdet durch Staatsinsolvenzen und Bankenkrisen in den Schuldnerländern. Deshalb steht jede deutsche Regierung nun unter dem Zwang, den Euro um fast jeden Preis verteidigen zu müssen.

Abbildung 1: Realer effektiver Wechselkurs gegenüber der Eurozone

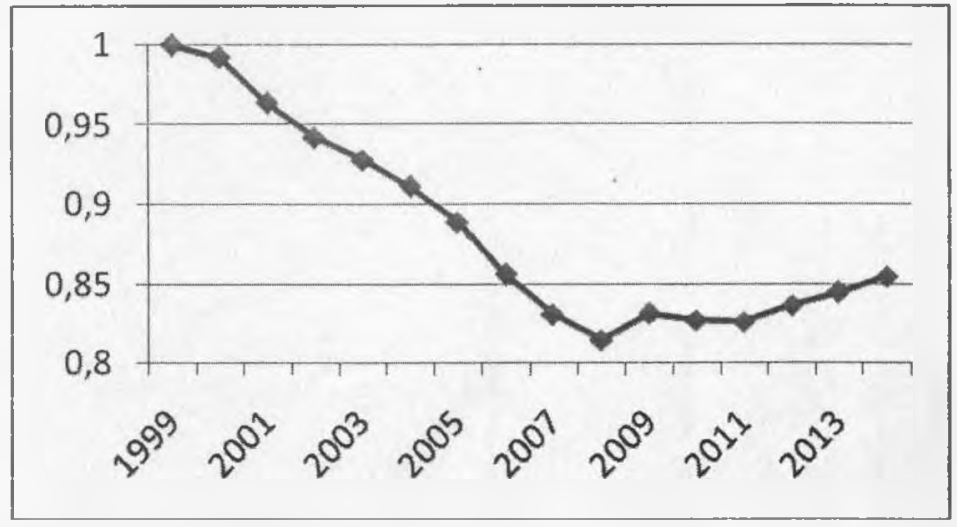

Quelle: Eurostat, Basis: Lohnstückkosten

Abbildung 2: Exporte als Anteil am BIP

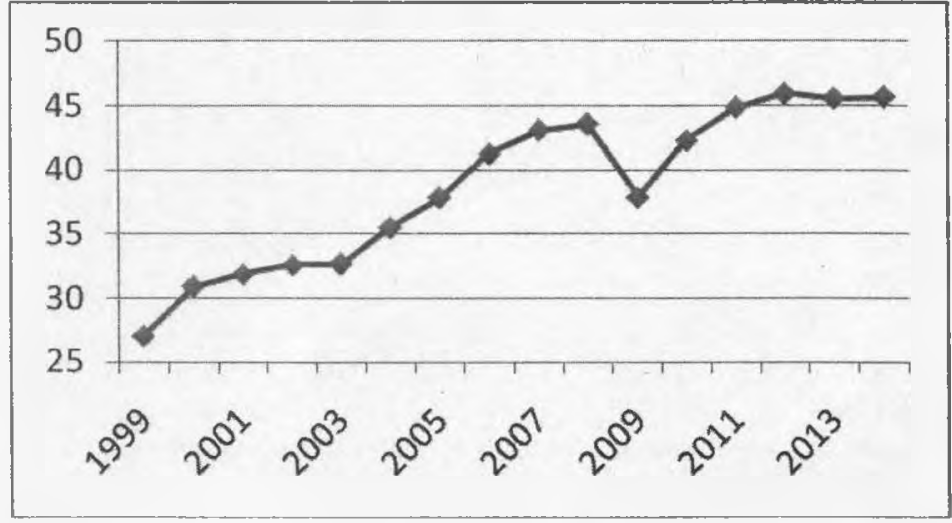

Quelle: Eurostat 
Abbildung 3: Netto-Auslandsposition als Anteil am BIP

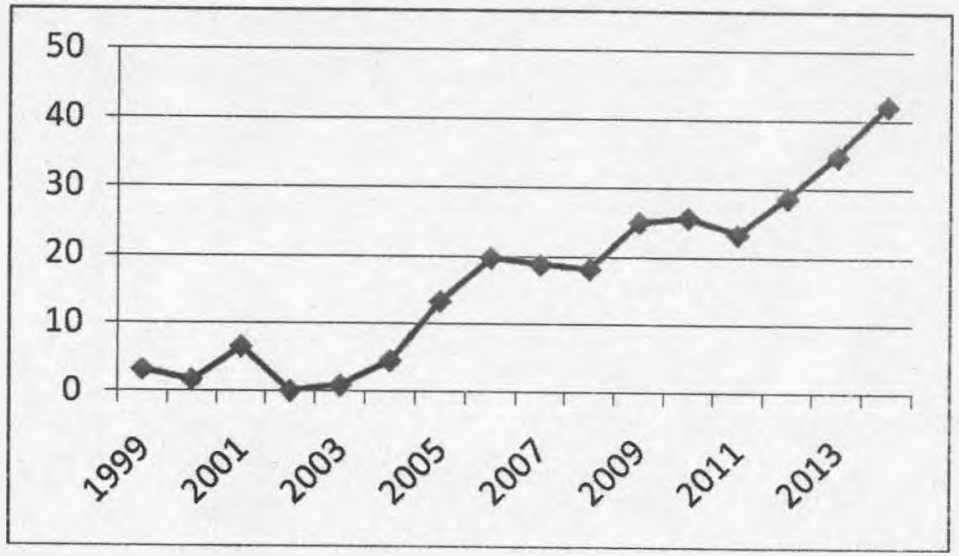

Quelle: Eurostat

\section{Aber das auf Deutschland zugeschnittene Euro-Regime passt nicht überall}

In der weltweiten Banken- und Wirtschaftskrise von 2008-09 waren die Staatsdefizite überall gestiegen. Aber zu einer Staatsschulden-Krise war es nur in der Eurozone - also in Ländern ohne eigene Wähnung - und auch da nur in Ländern mit hohen Leistungsbilanzdefiziten gekommen, die aufgrund ihrer Abhängigkeit vom internationalen Kapitalmarkt verwundbar waren. Erstes Ziel des derzeitigen Euro-Regimes war und ist es deshalb, Leistungsbilanzdefizite abzubauen und damit eine Wiederkehr der Eurokrise zu verhindern. Seine stringenten Austeritäts- und Reformauflagen für Länder, die Rettungskredite des Europäischen Stabilitätsmechanismus (ESM) in Anspruch nehmen müssen, und ebenso die dauerhafte Stabilitätsordnung der Six-Pack und Two-Pack-Verordnungen, des "Europäischen Semesters" und des Fiskalpakts sollen und können die Binnennachfrage beschränken und die Lohnkosten senken. Sie beschränken damit die Importe, verbilligen die Exporte und dienen so dem Abbau von Leistungsbilanzdefiziten und der Vermeidung neuer Staatsschuldenkrisen, die den Euro gefährden könnten.

Die von der deutschen Seite geprägte Euro-Politik passt auch gut zu Deutschland und einigen ähnlich exportstarken Mitgliedsländern, zu denen übrigens auch Irland gehört. Thr Exportsektor ist groß und leistungsfähig genug, um durch Kostensenkung und steigende Exportnachfrage auch ein exportorientiertes Wachs- 
tum der Gesamtwirtschaft zu erzeugen. Aber die gegenwärtige Euro-Politik bietet keine Gewähr dafür, dass auf diesem Wege alle Mitglieder der Eurozone auch die in der Krise erlittenen BIP- und Beschäftigungsverluste ausgleichen könnten. Und das gilt auch für den Fall künftiger Rezessionen.

Anders als Deutschland oder Irland können nämlich Länder mit einem relativ kleinen Exportsektor, zu denen beispielsweise auch Großbritannien und Frankreich gehören, nicht hoffen, durch bloße Senkung der Produktionskosten über höhere Exporte ihre Krisen zu überwinden. Im Gegenteil: Länder, in denen der weitaus größere Teil der Wirtschaft von der Binnennachfrage abhängt, wird diese durch Austeritätspolitik noch weiter geschwächt, und weil ihr Exportsektor klein ist, reicht die durch Lohnsenkung im besten Fall erreichbare höhere Exportnachfrage nicht aus, um den Niedergang auszugleichen. Sie werden also durch das Euro-Regime noch tiefer in die Krise getrieben und dann auf jeweils tieferem Niveau zu wirtschaftlicher Stagnation und Unterbeschäftigung verurteilt.

An dieser Lage kann offenbar auch die extrem expansive Geldpolitik der EZB kaum etwas ändern. Sie bestätigt vielmehr die schon zu Zeiten der Bundesbank gewonnene Einsicht, dass eine harte monetäre Restriktion zwar jede Konjunktur abwürgen kann, während in der Stagnation die bloße monetäre Expansion nicht ausreicht, um - mit Karl Schiller gesprochen - ,die Pferde zum Saufen zu zwingen".

So haben zwar alle Euroländer, die nach 2010 Rettungskredite in Anspruch genommen haben, inzwischen durch rückläufige Importe und leicht steigende Exporte ihre Leistungsbilanz-Defizite und die reale Überbewertung des Wechselkurses abgebaut. Aber allenfalls Irland hat die Chance, auf diesem Weg schließlich auch die Folgen der Krise zu überwinden. In Griechenland, Portugal, Spanien und auch in Italien dagegen ist der Anteil des Exportsektors am BIP zu klein, um allein durch höhere Exportnachfrage die Gesamtwirtschaft zu sanieren. In diesen Ländern ist deshalb die Beschäftigungsquote nach dem tiefen Einbruch in der Krise von 2009 auch unter dem Rettungsschirm noch weiter gefallen. In Deutschland dagegen stieg sie zwischen 2003und 2014 kontinuierlich von $64,6 \%$ auf den hier noch niemals zuvor erreichten Spitzenwert von $73,8 \%$.

Das absehbare Ergebnis der vom Euro-Regime durchgesetzten Kombination von fiskalischer Austerität und Lohndämpfung ist also eine immer tiefere Spaltung der Eurozone in eine Gruppe exportstarker und vom real unterbewerteten Wechselkurs begünstigter Länder und eine andere Gruppe von Ländern, deren von der 
Binnennachfrage abhängige Wirtschaft unter dem Euro-Regime nicht expandieren kann und von jeder künftigen Rezession noch tiefer in die Krise getrieben wird.

\section{Die deutsche Hegemonie wird zum Problem}

Dieses asymmetrisch wirkende Regime ist, nachdem man in der Panik der drohenden Eurokrise im Frïhjahr 2010 auf die Einhaltung der No-Bailout-Regeln des Maastricht-Vertrags verzichtet hatte, von der deutschen Politik gestaltet worden. Es sollte und soll emeut steigende Leistungsbilanz-Defizite und damit die Wiederkehr der Eurokrise verhindem. Zugleich entspricht es aber auch offensichtlich den ökonomischen Interessen Deutschlands, und seine Regeln sind unter den besonderen Bedingungen des „deutschen Modells" hierzulande ohne Konflikt einzuhalten. In anderen Ländern der Eurozone dagegen erzeugen sie Stagnation, Unterbeschäftigung und soziale Krisen. Sie sind deshalb schwer einzuhalten und politisch höchst umstritten. Ihre strikte Durchsetzung wird bisher vor allem durch den - auf die Vetomacht des größten „Zahlerlandes“ gestützten - Einfluss der deutschen Regierung in den intergouvernementalen Verhandlungen der Euro-Gruppe, des ECOFIN-Rats und in den Gipfeltreffen gesichert.

Aber je härter und sichtbarer die deutsche Position hier durchgesetzt wird, desto mehr verbreiten sich die Zweifel, ob damit neben dem deutschen auch das gemeinsame Interesse der Eurozone gefördert werde. Immer lauter werden deshalb politische Forderungen, die nicht nur Korrekturen im Einzelnen, sondern einen fundamentalen Richtungswechsel der Euro-Politik verlangen. Und es ist jedenfalls nicht sicher, dass die deutsche Politik in der Lage sein wird, solche Forderungen dauerhaft abzuwehren, ohne zugleich an Einfluss auf anderen Politikfeldern zu verlieren, in denen sie statt auf Vetomacht auf die freiwillige Zustimmung oder (beispielsweise in der Flüchtlingskrise) gar auf die Solidarität ihrer europäischen Partner angewiesen wäre.

Wenn aber hinhaltender Widerstand nicht länger genügen sollte, stünde die deutsche Euro-Politik vor einem Dilemma: Auch ihre Kritiker wollen ja an der gemeinsamen Währung festhalten, und unter dieser Voraussetzung ist die deutsche Position, obwohl sie deutschen Interessen zugute kommt, auch aus der Perspektive der Währungsunion und ihrer Stabilität in der Sache begründet. Jedenfalls wären die zumeist geforderten Korrekturen der Euro-Politik fast durchweg entweder ökonomisch unplausibel oder sogar schädlich. Bloßes Nachgeben wäre also keine Lösung. 


\section{Von der Lockerung der Sparzwänge zur Transferunion}

Ökonomisch am wenigsten unplausibel erscheint der auch von der Kommission geforderte Abbau der immer noch steigenden Überschüsse der deutschen Leistungsbilanz. Sie verletzen in der Tat fortwährend die Regeln der im Six-Pack beschlossenen EU-Verordnung über die Vermeidung makroökonomischer Ungleichgewichte. Freilich hat die Kommission diesen Verstoß zwar immer wieder gerügt, aber bisher auf Durchsetzung verzichtet - sei es aus Rücksicht auf die deutsche Verhandlungsmacht oder aufgrund eigener Zweifel an der Wirkung möglicher Sanktionen. Schließlich könnte die deutsche Regierung ja weder die Gewerkschaften zum Streik für höhere Löhne noch die Verbraucher zu höherer Verschuldung zwingen; und auch höhere Staatsausgaben würden inzwischen wohl eher deutsche Importe aus Asien denn aus Portugal und Griechenland begünstigen.

Im Vergleich dazu würde die nicht nur von den betroffenen Regierungen erhobene Forderung nach einer Lockerung der Sparzwänge in den Defizitländern dem primären Ziel einer Stabilisierung des Euro zuwiderlaufen. Zwar könnte eine fiskalische Expansion die Binnennachfrage und das Wachstum steigern. Aber damit würden ja nicht nur die Schulden der Staaten, sondern auch die eben abgebauten Defizite der Leistungsbilanzen und die Auslandsschulden der Wirtschaft wieder steigen. Mit der emeut wachsenden Abhängigkeit vom internationalen Kapitalmarkt würde dann auch das Risiko spekulativer Attacken auf die Solvenz der Defizit-Staaten und damit auf die Stabilität des Euro wieder zunehmen.

Die von den Krisenländern geforderte Lockerung der Sparzwänge wäre ökonomisch also nur dann sinnvoll, wenn die fiskalische Reflation zur Erhöhung der Binnennachfrage nicht durch Kredite finanziert würde. Dann könnten Verbrauch und Investitionen in der Binnenwirtschaft wieder wachsen, und soweit die dann steigenden Leistungsbilanzdefizite durch Transfers ausgeglichen werden, wären sie auch kein Risiko für den Euro. Dieser Logik entsprechen die meisten der international diskutierten Vorschläge zur Überwindung der wirtschaftlichen und sozialen Malaise der Eurozone durch Reformen des Euro-Regimes - von der Fiskalunion und dem europäischen Finanzminister, einem europäischen Marshallplan oder einer europäischen Arbeitslosenversicherung, bis hin zur bloßen Ausweitung der aus dem EU-Haushalt zu finanzierenden Kohäsionsprogramme. In der Sache geht es dabei immer um eine fiskalische Umverteilung zwischen den Überschuss- und den Krisenländern, die in diesen eine nicht- 
kreditfinanzierte Ausweitung der Konsum- und Investitionsnachfrage und damit höheres Wachstum und mehr Beschäftigung bewirken soll.

Aus der Sicht der strukturell exportschwachen Euro-Länder erscheint dies in der Tat als schlüssige Antwort auf die ökonomische Spaltung der Eurozone. Dass die deutsche Politik sich dagegen im eigenen Interesse wehrt, ist ebenso plausibel: Die Überschüsse der Leistungsbilanz stehen ja nicht zur Verfügung der Regierung; ihnen stehen private Rechtstitel gegenüber. Die Transfers müssten also wie alle zusätzlichen Staatsausgaben durch höhere Steuern, Kürzungen an anderer Stelle oder durch Kredite finanziert werden. Die politischen Kosten lägen also auf der Hand. Vor allem aber fehlt der deutschen Politik auch in der Sache die Hoffnung, man könnte unter den Bedingungen einer Währungsunion in einer wirtschaftsschwachen Region durch Transfers selbsttragendes Wachstum erzeugen. Der Versuch, ein solches Ergebnis nach der deutschen Vereinigung in Ostdeutschland zu erreichen, hat inzwischen West-Ost-Transfers im Umfang von ca. 1,7 Billionen Euro erfordert. Aber nach einem Vierteljahrhundert intensiver Förderung unter nahezu optimalen institutionellen und politischen Bedingungen liegt das in Ostdeutschland erzeugte Bruttoinlandsprodukt immer noch erst bei zwei Dritteln des westdeutschen. Und nach sechs Jahrzehnten hoher Nord-SüdTransfers ist auch der Mezzogiorno immer noch das Armenhaus Italiens.

Der Versuch, die ökonomische Spaltung der Eurozone durch Transfers zu überwinden, könnte also statt des erhofften Wiederaufstiegs der exportschwachen Länder deren dauerhafte Subventionsabhängigkeit bewirken. Zugleich aber wäre zu erwarten, dass die fiskalischen Belastungen die Wirtschaft der Geberländer ebenso schwächen werden, wie dies nach der deutschen Vereinigung in Westdeutschland der Fall war. Die ökonomische Spaltung der Eurozone würde zwar gemildert, wenn die Leistungsbilanzdefizite der einen Seite durch die Transfers der anderen Seite finanziert würden. Aber im Ergebnis könnte dies auf die gemeinsame Stagnation aller Mitglieder der Eurozone hinauslaufen. Die deutsche Politik jedenfalls scheint von dieser Diagnose auszugehen.

\section{Differenzierte Integration?}

Was aber bleibt, wenn die deutsche Euro-Politik immer stärker unter Druck gesetzt wird, während alle ernsthaft vertretenen Forderungen auf die aus deutscher Sicht politisch unerwünschte und ökonomisch problematische Transferunion hinauslaufen? Dann könnte hinhaltender Widerstand so wenig helfen wie das alte deutsche Ablenkungsmanöver, die vorderhand unerreichbare Politische Union oder gar die Vereinigten Staaten von Europa zu fordern. Helfen könnten 
aber vielleicht deutsche Initiativen, welche die grundlegenden Mängel der Währungsunion und die ökonomische Spaltung der Eurozone nicht leugnen und auf dieser Grundlage nach Lösungen suchen, die nicht nur deutschen Interessen dienen.

Einen möglichen Ausweg hat Schäuble selbst im vergangen Spätsommer angedeutet, als er den Grexit als ultimative Drohung im Showdown über den Griechen-Kredit ins Spiel brachte. Dafuir gab es aber offenbar nicht einmal in Berlin genügend Unterstützung. Dennoch lohnt es sich, die Implikationen des angedeuteten Vorschlags zu überdenken. Sie entsprechen in interessanten Aspekten der Logik des berühmten Schäuble-Lamers-Papiers vom September 1994. Dort ging es um „flexible Methoden der Integration", um in einer politisch und ökonomisch zunehmend heterogenen Europäischen Union ein gemeinsam handlungsfähiges „Kemeuropa“ in die Lage zu versetzen, die Integration mit dem Ziel der Politischen Union voranzutreiben. In diesem Kontext sollte die Währungsunion „die es voraussichtlich zunächst nur in einem kleineren Kreis geben“ wird - der „harte Kern der Politischen Union“ werden, dessen Mitglieder schon jetzt eine,gemeinsame, gleichgerichtete" Geldpolitik, Fiskalpolitik, Wirtschafts- und Sozialpolitik verfolgen sollten.

Daran gemessen muss die gegenwärtige Eurozone als eklatante Fehlentwicklung erscheinen. Sie ist viel zu groß und zu heterogen geworden, als dass ihre Mitglieder die gemeinsame Politik aus freien Stücken und mit demokratischer Legitimation beschließen könnten. Diese muss deshalb oktroyiert und mit angedrohtem Staatsbankrott und Sanktionen erzwungen werden. Und wo sie durchgesetzt wird, befördert sie nicht die politische Integration, sondern mit der ökonomischen auch die politische Spaltung Europas. Der Mitverfasser des SchäubleLamers-Papiers muss darin ein Unglück sehen. Der angedrohte Grexit kann deshalb auch als Schritt auf dem Weg zur Korrektur einer historischen Fehlentwicklung interpretiert werden. Wenn Austritte möglich werden, dann könnte eine kompaktere und homogenere Währungsunion schließlich doch noch den harten Kern einer künftigen Politischen Union bilden.

Freilich reproduziert die Drohung auch das Defizit, das der Wirkung des Schäuble-Lamers-Papiers außerhalb Deutschlands entgegen stand: In seiner Fixierung auf den „Kern“ ignorierte es die Interessen der Länder, die aus deutscher Sicht nicht dazu gehören sollten. Sie konnten allenfalls hoffen, bei ernstem Bemühen später beitreten zu dürfen. Es gab in dem Papier jedenfalls kein Konzept für die Anerkennung und faire Berücksichtigung der legitimen Interessen der „Periphe-

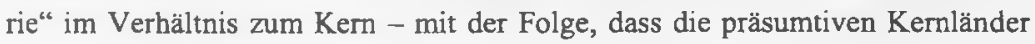


dann auch politisch nicht in der Lage waren, die Beitrittsforderungen zu vieler Peripheriestaaten politisch abzuweisen.

Qffenbar hatte Schäuble bei seiner Grexit-Drohung aber nicht nur den (rechtlich unmöglichen) bloßen Rauswurf Griechenlands im Sinn. Denn nach Auskunft von Yanis Varoufakis bot er im privaten Gespräch auch Übergangshilfen im Falle eines (rechtlich allenfalls möglichen) freiwilligen Austritts an. Das allein hätte freilich auch bei emsthafter Diskussion nicht ausgereicht, um die geostrategischen Bedenken der Amerikaner und der NATO sowie die Angst der Griechen vor der Isolation in Europa, vor den Fähmissen der Währungsmärkte und vor der Gefahr selbst verursachter Inflationsabwertungs-Spiralen zu überwinden.

Aber hätte die Diskussion nicht anders ausgehen können, wenn nicht nur die vage Andeutung von Übergangshilfen, sondern klare und faire Regeln für den Austritt aus der Währungsunion und seine Folgen in Geltung gewesen wären? Dazu bedürfte es nicht nur der seit Langem geforderten Konkursordnung für überschuldete Euro-Staaten. Geregelt werden müssten auch die Voraussetzungen und Verfahren des Austritts und die Hilfen für den Übergang sowie vor allem die künftigen Beziehungen zwischen dem ausgetretenen Staat und der sich weiter vertiefenden Europäischen Währungsunion.

\section{Ein Modell gibt es schon}

Tatsächlich ist ein Teil einer solchen Ordnung schon jetzt vorhanden: Der Europäische Wechselkursmechanismus II (WKM II) entstand 1999 beim Übergang vom 1979 eingerichteten Europäischen Währungssystem (EWS) zur Europäischen Währungsunion (EWU). Er diente dazu, die Währung künftiger Beitrittsländer schrittweise an den Euro heranzuführen. Nachdem aber inzwischen auch die baltischen Kandidaten die volle Mitgliedschaft erreicht haben, gilt der WKM II derzeit nur noch für Dänemark, das den Beitritt jedoch gar nicht anstrebt. Diese Struktur könnte im Prinzip auch das Verhältnis zwischen dem Euro und der Währung von Ländern regeln, die zwar aus der Währungsunion ausgetreten sind, aber dennoch in einer geregelten Beziehung zur Währungsunion bleiben wollen und sollen.

Im Kem geht es hier um folgende Regeln: Der Wechselkurs der Währung eines WKM-Staates wird im Verhältnis zum Euro festgelegt. Der Staat behält die Kompetenz für die Geld- und Fiskalpolitik und sollte diese so einsetzen, dass der Kurs seiner Währung die vereinbarte Parität nicht um mehr als $15 \%$ über- oder unterschreitet. Nähert sich der Wechselkurs dieser Grenze, so sind die EZB und 
die nationale Zentralbank zur Intervention auf dem Währungsmarkt verpflichtet. Zur Überbrückung kurzfristiger Zahlungsbilanzdefizite sind auch Währungskredite der EZB möglich. Reichen diese Instrumente zur Stabilisierung des Wechselkurses nicht aus, so kann eine Aufwertung oder Abwertung vereinbart werden.

Im Gegensatz zur Mitgliedschaft in der Währungsunion läge der entscheidende Vorteil für den WKM-Staat in der Möglichkeit einer auf die Lage der nationalen Wirtschaft bezogenen Geld- und Fiskalpolitik. Im Vergleich zu einer völlig eigenständigen nationalen Währung wäre diese Autonomie freilich durch die Pflicht zur Verteidigung des Euro-Wechselkurses begrenzt. Aber diesem Nachteil stehen zwei wichtige Vorteile gegenüber: Mitglieder des WKM wären gegen spekulative Attacken auf ihren Wechselkurs durch die faktisch unbegrenzte „Feuerkraft“ der EZB-Interventionen geschützt. Zudem sollten die zur Stabilisierung des Wechselkurses notwendige Kooperation mit der EZB und die für eine Änderung der Paritäten notwendigen Vereinbarungen auch unkontrollierbare Inflations-Abwertungsspiralen und kompetitive Abwertungen verhindern.

Freilich ist der WKM II in seiner bisherigen Form als Einbahnstraße auf dem Weg zur Vollmitgliedschaft in der Währungsunion konzipiert. Um für eine Reform des Euro-Regimes nutzbar zu werden, müsste der Übergang in beiden Richtungen ermöglicht und geregelt werden. Sofern mit dem Austritt aus der EWU eine unmittelbare Abwertung verbunden wäre, sollten auch deren Folgen für laufende Verträge und Verpflichtungen vorab geregelt werden. Das wird schwierig sein. Aber da im EWS zwischen 1979 und 1999 insgesamt 62 Wechselkursänderungen bewältigt wurden, gibt es auch einen reichen Fundus praktischer Erfahrungen, die bei einer generellen Regelung genutzt werden können. Hätte ein solches Regelwerk schon im Frühjahr 2010 oder im Sommer 2015 existiert, dann wäre es im Falle Griechenland eben nicht um die Gefahr eines unkontrollierten Zusammenbruchs der Währungsunion gegangen, dessen Antizipation im Frühjahr 2010 die Anwendung der No-Bailout-Klausel und vielleicht auch noch 2015 den Grexit verhinderte, sondern um die allerdings folgenreiche und schwierige Anwendung einer generellen Regel auf den Einzelfall.

Eine solche Reform könnte freilich nicht unter dem Druck einer akuten Eurokrise ausgehandelt werden. Aber vielleicht ist die gegenwärtige Ruhe nach dem Grexit-Konflikt für Verhandlungen zu nutzen. Die Initiative dazu müsste wohl von Berlin ausgehen, das mit der Eröffnung von Austrittsmöglichkeiten dem aiten Ziel wieder näher käme, die Währungsunion zum „,harten Kern" einer europäischen Politischen Union weiterzuentwickeln. Aber sie hätte politisch nur dann eine Chance, wenn sie - im Gegensatz zum Schäuble-Lamers-Konzept - 
auch eine europäische Perspektive für jene Länder eröffnet, die diesem Kern nicht angehören wollen oder können. Deshalb genügte es nicht, lediglich Regeln für den Austritt aus dem Euro vorzuschlagen. Nötig wären vielmehr Regeln für ein zweistufiges monetäres Regime, das eine stabilisierende Koppelung zwischen dem Euro und der Währung europäischer Länder vorsieht, die zwar nicht der Währungsunion, wohl aber einem europäischen Währungsverbund angehören wollen. Eine Grundstruktur, auf der man weiterbauen könnte, liegt dafür schon bereit. 